\title{
Policy-Aware Visualization of Internet Dynamics
}

\author{
Luca Cittadini, Tiziana Refice, Alessio Campisano, \\ Giuseppe Di Battista, and Claudio Sasso \\ Dipartimento di Informatica e Automazione - Università di Roma Tre \\ $\{$ ratm,refice,gdb\}@dia.uniroma3.it, \\ \{alessio.campisano, claudio.sasso\}@gmail.com
}

\section{Why Visualizing Inter-domain Routing Dynamics}

The Internet can be represented as a graph of Autonomous Systems (ASes). Each AS dynamically selects the $(A S$-) paths to reach destinations on the Internet, according to inter-AS customer-provider relationships. Such relationships define a hierarchy of all the ASes.

The Internet is renowned to be highly dynamic as AS-paths to any destination may frequently change (from an old path to a new path). Such AS-path changes may impact the Internet operation and are usually debugged manually. Figure 1(a) shows the AS-paths (valid at a specific time) from a set of ASes to a specific destination, as displayed by BGPlay 2. After a few seconds the state of the network can be significantly different. Due to the enormous amount of AS-path changes occurring in a short time period, it is very difficult to spot them and, thus, to locate their root causes. Hence, effectively visualizing an AS-path change can significantly help understand the Internet dynamics.

(a)

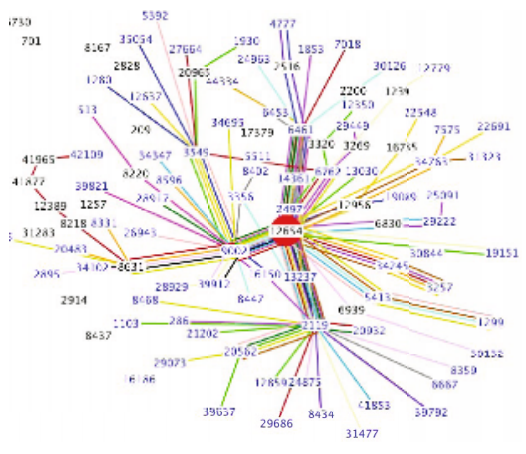

(b)

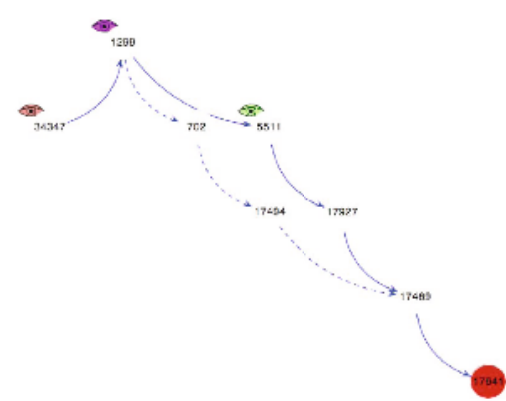

Fig. 1. (a) AS-paths towards a specific destination. Each number represents an AS. The red node is the destination. (b) An AS-path change displayed by BGPATH. Solid edges belong to the new path, dashed edges belong to the old path. 


\section{How-To Visualize Internet Dynamics to Support Analysis of Network Events}

We propose to focus the visualization on a single AS-path change and to display it on the customer-provider hierarchy, as [6] shows that network events located at different levels of the hierarchy have usually significantly different impact on the Internet.

We now describe our algorithm to draw an AS-path change. First, we assign customer-provider relationships to the links of both old and new AS-paths, and we direct the links from providers to customers. As shown by [5], we can then classify ASes of both paths according to the valley-free property as follows:

type 1 : nodes in the "uphill" portion of the old path

type 2 : nodes in the "uphill" portion of the new path

type 3 : nodes in the "downhill" portion of the old path

type 4 : nodes in the "downhill" portion of the new path

We assign vertical coordinates using a topological sort of the graph. We then compute the horizontal coordinates. Namely, we first split edges spanning over multiple vertical layers by adding extra nodes and edges. Further, we add extra edges between nodes on the same layer, from nodes with lower type values to nodes with higher values. Finally, the topological sort of this augmented graph provides us with the horizontal coordinates, such that nodes in the new path are placed right of nodes in the old path, according to the common intuition of time flowing left-to-right.

In 34 we detail how our visualization paradigm supports the analysis of network events. We also developed a publicly available tool, BGPATH [1], which visualizes a user-specified AS-path change according to the approach described above and provides useful information to help identify its root cause. Figure 1(b) shows how BGPATH displays a sample AS-path change.

\section{References}

1. BGPath, http://nero.dia.uniroma3.it/rca/

2. BGPlay, http://www.ris.ripe.net/bgplay/

3. Campisano, A., Cittadini, L., Di Battista, G., Refice, T., Sasso, C.: Tracking Back the Root Cause of a Path Change in Interdomain Routing. In: IEEE/IFIP NOMS (2008)

4. Cittadini, L., Refice, T., Campisano, A., Di Battista, G., Sasso, C.: Measuring and Visualizing Interdomain Routing Dynamics with BGPath. In: IEEE ISCC (2008)

5. Gao, L.: On Inferring Autonomous System Relationships in the Internet. IEEE/ACM Transactions on Networking (2001)

6. Zhao, X., Zhang, B., Terzis, A., Massey, D., Zhang, L.: The Impacts of Link Failure Location on Routing Dynamics: A Formal Analysis. In: ACM SIGCOMM Asia Workshop (2005) 